Pacific Journal of Mathematics

A CHARACTERIZATION THEOREM FOR COMPACT UNIONS
OF TWO STARSHAPED SETS IN $R^{3}$ 


\title{
A CHARACTERIZATION THEOREM FOR COMPACT UNIONS OF TWO STARSHAPED SETS IN $R^{3}$
}

\author{
MARILYN BREEN
}

Set $S$ in $R^{d}$ has property $P_{k}$ if and only if $S$ is a finite union of $d$-polytopes and for every finite set $F$ in bdry $S$ there exist points $c_{1}, \ldots, c_{k}$ (depending on $F$ ) such that each point of $F$ is clearly visible via $S$ from at least one $c_{i}, 1 \leq i \leq k$. The following results are established.

(1) Let $S \subseteq R^{3}$. If $S$ satisfies property $P_{2}$, then $S$ is a union of two starshaped sets.

(2) Let $S \subseteq R^{d}, d \geq 3$. If $S$ is a compact union of $k$ starshaped sets, then there exists a sequence $\left\{S_{j}\right\}$ converging to $S$ (relative to the Hausdorff metric) such that each set $S_{j}$ satisfies property $P_{k}$.

When $d=3$ and $k=2$, the converse of (2) above holds as well, yielding a characterization theorem for compact unions of two starshaped sets in $R^{3}$.

1. Introduction. We begin with some definitions. Let $S$ be a subset of $R^{d}$. Hyperplane $H$ is said to support $S$ locally at boundary point $s$ of $S$ if and only if $s \in H$ and there is some neighborhood $N$ of $s$ such that $N \cap S$ lies in one of the closed halfspaces determined by $H$. Point $s$ in $S$ is called a point of local convexity of $S$ if and only if there is some neighborhood $N$ of $s$ such that $N \cap S$ is convex. If $S$ fails to be locally convex at $q$ in $S$, then $q$ is called a point of local nonconvexity (lnc point) of $S$. For points $x$ and $y$ in $S$, we say $x$ sees $y$ via $S$ ( $x$ is visible from $y$ via $S$ ) if and only if the segment $[x, y]$ lies in $S$. Similarly, $x$ is clearly visible from $y$ via $S$ if and only if there is some neighborhood $N$ of $x$ such that $y$ sees via $S$ each point of $N \cap S$. Set $S$ is locally starshaped at point $x$ of $S$ if and only if there is some neighborhood $N$ of $x$ such that $x$ sees via $S$ each point of $N \cap S$. Finally, set $S$ is starshaped if and only if there is some point $p$ in $S$ such that $p$ sees via $S$ each point of $S$, and the set of all such points $p$ is called the (convex) kernel of $S$.

A well-known theorem of Krasnosel'skii [3] states that if $S$ is a nonempty compact set in $R^{d}, S$ is starshaped if and only if every $d+1$ points of $S$ are visible via $S$ from a common point. Moreover, "points of $S$ " may be replaced by "boundary points of $S$ " to produce a stronger result. In [1], the concept of clear visibility, together with work by Lawrence, Hare, and Kenelly [4], were used to obtain the following 
Krasnosel'skii-type theorem for unions of two starshaped sets in the plane: Let $S$ be a compact nonempty set in $R^{2}$, and assume that for each finite set $F$ in the boundary of $S$ there exist points $c, d$ (depending on $F$ ) such that each point of $F$ is clearly visible via $S$ from at least one of $c, d$. Then $S$ is a union of two starshaped sets.

In this paper, an analogous result is proved for set $S$ in $R^{3}$, where $S$ satisfies the additional hypothesis of being a finite union of polytopes. Furthermore, while not every compact union $F$ of two starshaped sets in $R^{3}$ satisfies this hypothesis, $F$ will be the limit (relative to the Hausdorff metric) for a sequence whose members do satisfy it. This in turn leads to a characterization theorem for compact unions of two starshaped sets in $R^{3}$.

The following terminology will be used throughout the paper: Conv $S$, $\operatorname{cl} S$, int $S$, rel int $S$, bdry $S$, rel bdry $S$, and $\operatorname{ker} S$ will denote the convex hull, closure, interior, relative interior, boundary, relative boundary, and kernel, respectively, for set $S$. The distance from point $x$ to point $y$ will be denoted $\operatorname{dist}(x, y)$. For distinct points $x$ and $y, L(x, y)$ will be the line determined by $x$ and $y$, while $R(x, y)$ will be the ray emanating from $x$ through $y$. For $x \in S, A_{z}$ will represent $\{x: z$ is clearly visible via $S$ from $x\}$. The reader is referred to Valentine [7] and to Lay [5] for a discussion of these concepts and to Nadler [6] for information on the Hausdorff metric.

2. The results. The following definition will be helpful.

Definition 1. Let $S \subseteq R^{d}$. We say that $S$ has property $P_{k}$ if and only if $S$ is a finite union of $d$-polytopes and for every finite set $F \subseteq$ bdry $S$ there exist points $c_{1}, \ldots, c_{k}$ (depending on $F$ ) such that each point of $F$ is clearly visible via $S$ from at least one $c_{i}, 1 \leq i \leq k$.

Several lemmas will be needed to prove Theorem 1 . The first of these is a variation of $[2$, Lemma 2$]$.

LEMMA 1. Let $S \subseteq R^{d}, z \in S$, and assume that $S$ is locally starshaped at $z$. If $p \in \operatorname{conv} A_{z}$ and $p \neq z$, then there exists some point $p^{\prime} \in[p, z)$ such that $p^{\prime} \in A_{z}$.

Proof. As in [2, Lemma 2], use Carathéodory's theorem to select a set of $d+1$ or fewer points $p_{1}, \ldots, p_{k}$ in $A_{z}$ with $p \in \operatorname{conv}\left\{p_{1}, \ldots, p_{k}\right\}$. Say $p=\Sigma\left\{\lambda_{i} p_{i}: 1 \leq i \leq k\right\}$, where $0 \leq \lambda_{i} \leq 1$ and $\Sigma\left\{\lambda_{i}: 1 \leq i \leq k\right\}=1$. Observe that for any $0 \leq \mu \leq 1$, point $\mu z+(1-\mu) p$ on $[z, p]$ is a convex conbination of the points $\mu z+(1-\mu) p_{i}, 1 \leq i \leq k$. Also $\mu z+$ $(1-\mu) p_{i} \in\left[z, p_{i}\right], 1 \leq i \leq k$. By the definition of locally starshaped, 
together with the definition of clear visibility, we may choose a spherical neighborhood $N$ of $z, p \notin N$, such that $z$ and each $p_{i}$ see via $S$ every point of $N \cap S$. We may choose $\mu_{0}, 0<\mu_{0}<1$ and $\mu_{0}$ sufficiently near 1 that each point $\mu_{0} z+\left(1-\mu_{0}\right) p_{i}=p_{i}^{\prime}$ belongs to $N$. Define

$$
\begin{aligned}
p^{\prime} & =\Sigma\left\{\lambda_{i} p_{i}^{\prime}: 1 \leq i \leq k\right\} \\
& =\mu_{0} z+\left(1-\mu_{0}\right) p \in \operatorname{conv}\left\{p_{1}^{\prime}, \ldots, p_{k}^{\prime}\right\} \cap(z, p) \cap N .
\end{aligned}
$$

We will show that $p^{\prime}$ satisfies the lemma. For $x \in N \cap S,[x, z] \subseteq N$ $\cap S, p_{1}$ sees $[x, z]$ via $S$, and hence $\operatorname{conv}\left\{p_{1}^{\prime}, x, z\right\} \subseteq N \cap S$. By an easy induction, $\operatorname{conv}\left\{p_{k}^{\prime}, \ldots, p_{1}^{\prime}, x, z\right\} \subseteq N \cap S$. Since $p^{\prime} \in \operatorname{conv}\left\{p_{k}^{\prime}, \ldots, p_{1}^{\prime}\right\}$, $\left[p^{\prime}, x\right] \subseteq S$. We conclude that $p^{\prime}$ sees via $S$ each point of $N \cap S$, $p^{\prime} \in A_{z}$, and Lemma 1 is established.

LeMma 2. Let $S$ be a closed set in $R^{d}$. Let $P$ be a plane in $R^{d}, B$ a component of $P \sim S$, with $S$ locally starshaped at $z \in$ bdry $B$. Assume that line $L$ in plane $P$ supports $B$ locally at $z$ and that $B \cap M$ is in the open halfplane $L_{1}$ determined by $L$ for an appropriate neighborhood $M$ of $z$. Then (conv $\left.A_{z}\right) \cap P \subseteq \mathrm{cl} L_{2}$, where $L_{2}$ is the opposite open halfplane determined by $L$.

Proof. Suppose on the contrary that there is some point $p \in$ (conv $\left.A_{z}\right) \cap P \cap L_{1}$, to obtain a contradiction. Then $p \neq z$, so by Lemma 1 there exist point $p^{\prime} \in[p, z)$ and convex neighborhood $N$ of $z$ such that $p^{\prime}$ sees via $S$ each point of $N \cap S$. For convenience of notation, assume that $N \subseteq M \subseteq P$.

By a simple geometric argument, we may choose a point $b \in B \cap N$ such that $R\left(p^{\prime}, b\right)$ meets $N \cap L$ at some point $w$. Since $B \cap N \subseteq B \cap M$ $\subseteq L_{1}, w \notin B$, so $(b, w]$ meets bdry $B$ at a point $c$. We have $c \in[b, w] \subseteq N$ and $c \in$ bdry $B \subseteq S$, so $c \in N \cap S$. Therefore, by our choice of $p^{\prime}$, $\left[p^{\prime}, c\right] \subseteq S$. Hence $b \in\left[p^{\prime}, c\right] \subseteq S$, impossible since $b \in B \subseteq P \sim S$. We have a contradiction, our supposition is false, and $\left(\operatorname{conv} A_{z}\right) \cap P \subseteq \operatorname{cl} L_{2}$. Thus Lemma 2 is proved.

LEMMA 3. Let $S$ be a compact set in $R^{3}$, and assume that $S$ is a finite union of polytopes. Let $P$ be a plane in $R^{3}$, with $b$ a bounded component of $P \sim S$. For $z$ a point of local convexity of $\mathrm{cl} B, z$ in edge $e \subseteq \operatorname{rel}$ bdry cl $B$, there exists a plane $H$ such that the following are true:

(1) $H \cap P$ is a line containing $e$.

(2) The two open halfspaces determined by $\mathrm{H}$ can be denoted $\mathrm{H}_{1}$ and $\mathrm{H}_{2}$ in such $a$ way that for $N$ any neighborhood of $z$ such that $(\operatorname{cl} B) \cap N$ is convex, $B \cap N$ lies in $H_{1}$ while $A_{z} \subseteq \mathrm{cl} H_{2}$. 
Proof. Notice that $S$ is locally starshaped at each of its points and that bdry $B$ is a closed polygonal curve in $P$. Let $J$ be a plane, $J \neq P$, such that $J$ contains edge $e$ of bdry $B$. If $N$ is any neighborhood of $z$ such that $(\mathrm{cl} B) \cap N$ is convex, then $J$ supports $(\operatorname{cl} B) \cap N$ at $e$, and $B \cap N$ necessarily lies in one of the open halfspaces $J_{1}$ determined by $J$. If $A_{z} \subseteq \operatorname{cl} J_{2}$, then $J$ satisfies the lemma. Otherwise, $A_{z} \cap J_{1} \neq \varnothing$.

For convenience of notation, let $P_{1}$ and $P_{2}$ denote distinct open halfspaces in $R^{3}$ determined by plane $P$, let $L=P \cap J$, and label the halfplanes in $P$ determined by $L$ so that $B \cap N \subseteq L_{1} \equiv J_{1} \cap P$. (See Figure 1.) Observe that conv $A_{z}$ is necessarily disjoint from one of $J_{1} \cap P_{1}$ or $J_{1} \cap P_{2}$, for otherwise $\left(\operatorname{conv} A_{z}\right) \cap J_{1} \cap P \equiv\left(\operatorname{conv} A_{z}\right) \cap L_{1} \cap P \neq \varnothing$, contradicting Lemma 2. Thus we may assume that $\left(\operatorname{conv} A_{z}\right) \cap J_{1} \cap P_{2}=$ $\varnothing$, and since $\left(\operatorname{conv} A_{z}\right) \cap L_{1}=\varnothing,\left(\operatorname{conv} A_{z}\right) \cap J_{1} \subseteq P_{1}$.

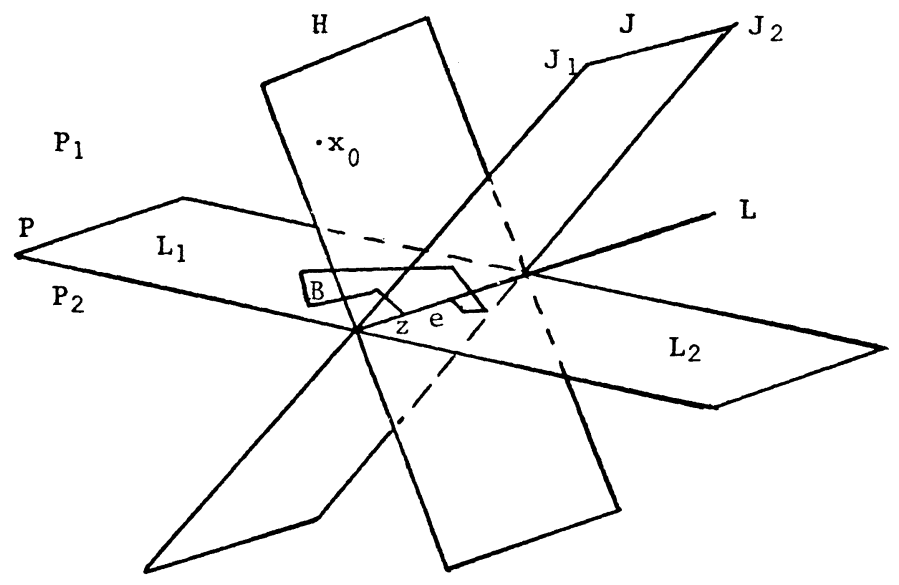

FIGURE 1

Examine the points of $A_{z} \cap J_{1} \subseteq P_{1}$. For $x \in A_{z} \cap J_{1}, x$ sees via $S$ a nondegenerate segment $s_{z}$ at $z$ contained in edge $e$, thus generating a planar set $T_{x} \equiv \operatorname{conv}\left(s_{x} \cup\{x\}\right)$. Since none of the $T_{x}$ sets lie in $P$, each determines with $\mathrm{cl} L_{1}$ an angle of positive measure $m(x)$. Define $m \equiv$ $\operatorname{glb}\left\{m(x): x \in A_{z} \cap J_{1}\right\}$. Since $S$ is a finite union of polytopes, the $T_{x}$ sets lie in a finite union of polytopes, each meeting edge $e$ in a nondegenerate segment at $z$, each contained in $P_{1} \cup L$. This forces $m$ to be positive. Using a standard argument, select sequence $\left\{x_{i}\right\}$ in $A_{z} \cap J_{1}$ so that $\left\{m\left(x_{1}\right)\right\}$ converges to $m$. Some subsequence of $\left\{x_{1}\right\}$ also converges, say to $x_{0}$. Moreover, the angle determined by $\operatorname{conv}\left(e \cup\left\{x_{0}\right)\right\}$ and $\mathrm{cl} L_{1}$ has measure $m$, and $x_{0} \in\left(\operatorname{cl} A_{z}\right) \cap J_{1} \subseteq P_{1}$. Let $H$ be the plane determined by conv $\left(e \cup\left\{x_{0}\right\}\right)$. Of course $H \cap P=L$. Furthermore, for an 
appropriate labeling of halfspaces determined by $H, L_{1} \subseteq H_{1}$ so $B \cap N$ $\subseteq H_{1}$.

It remains to show that $A_{z} \subseteq \mathrm{cl} H_{2}$. Suppose on the contrary that $y \in A_{z} \cap H_{1}$. If $y \in P_{1}$, then the angle $m$ chosen above would not be minimal. If $y \in P$, then $y \in A_{z} \cap P \cap L_{1}$, contradicting Lemma 2. If $y \in P_{2}$, then since $y \in P_{2} \cap H_{1}$ and $x_{0} \in P_{1} \cap H,\left[y, x_{0}\right]$ would meet $P \cap H_{1}=L_{1}$. Moreover, since $x_{0} \in \operatorname{cl} A_{z}$, there would be a point $x_{0}^{\prime} \in A_{z}$ sufficiently near $x_{0}$ that $\left[y, x_{0}^{\prime}\right]$ would meet $P \cap H_{1}=L_{1}$ also, say at point $w$. Then $w \in\left(\operatorname{conv} A_{z}\right) \cap P \cap L_{1}$, again contradicting Lemma 2. We conclude that $A_{z} \cap H_{1}=\varnothing$, and $A_{z} \subseteq \mathrm{cl} H_{2}$, finishing the proof of Lemma 3.

The final lemma follows immediately from [4, Theorem 1].

LeMma 4 (Lawrence, Hare, Kenelly Lemma). Let $S$ be a closed set in $R^{d}$. Assume that every finite set $F$ in bdry $S$ may be partitioned into two sets $F_{1}$ and $F_{2}$ such that each point of $F_{i}$ is clearly visible from a common point of $S$. Then bdry $S$ may be partitioned into two sets $S_{1}$ and $S_{2}$ such that for every finite set $F$ in bdry $S$, each point of $F \cap S_{l}$ is clearly visible from a common point of $S, i=1,2$.

We are ready to prove the following theorem.

THEOREM 1. Let $S \subseteq R^{3}$. If $S$ satisfies property $P_{2}$, then $S$ is a union of two starshaped sets.

Proof. Using Lemma 4, select a partition $S_{1}, S_{2}$ for bdry $S$ such that for every finite set $F$ in bdry $S$, each point of $F \cap S_{i}$ is clearly visible via $S$ from a common point. For $i=1,2$, define $\mathscr{T}_{i}=\left\{\operatorname{cl} A_{z}: z \in S_{i}\right\}$. Then each $\mathscr{T}_{i}$ is a collection of compact subsets of $S$. Moreover, by our choice of $S_{1}$ and $S_{2}$, each $\mathscr{T}_{i}$ has the finite intersection property. Hence $\cap\{: T$ in $\left.\mathscr{T}_{i}\right\} \neq \varnothing$, and we may select points $c$ and $d$ with $c \in \bigcap\left\{T: T\right.$ in $\left.\mathscr{T}_{1}\right\}$ and $d \in \bigcap\left\{T: T\right.$ in $\left.\mathscr{T}_{2}\right\}$. Observe that for $z \in$ bdry $S=S_{1} \cup S_{2}$, one of $c$ or $d$, say $c$, belongs to $\mathrm{cl} A_{z}$. Then $[c, z] \subseteq S$. We conclude that each boundary point of $S$ sees via $S$ either $c$ or $d$.

We will show that each point of $S$ sees via $S$ either $c$ or $d$. Portions of the argument will resemble the proof of [1, Theorem 1]. Let $x \in S$ and suppose on the contrary that neither $c$ nor $d$ sees $x$, to reach a contradiction. Certainly $x \notin\{c, d\}$, and by a previous observation. $x \in \operatorname{int} S$. As in [1, Theorem 1], choose the segment at $x$ in $S \cap L(c, x)$ having maximal length, and let $p$ and $q$ denote its endpoints, with the order of 


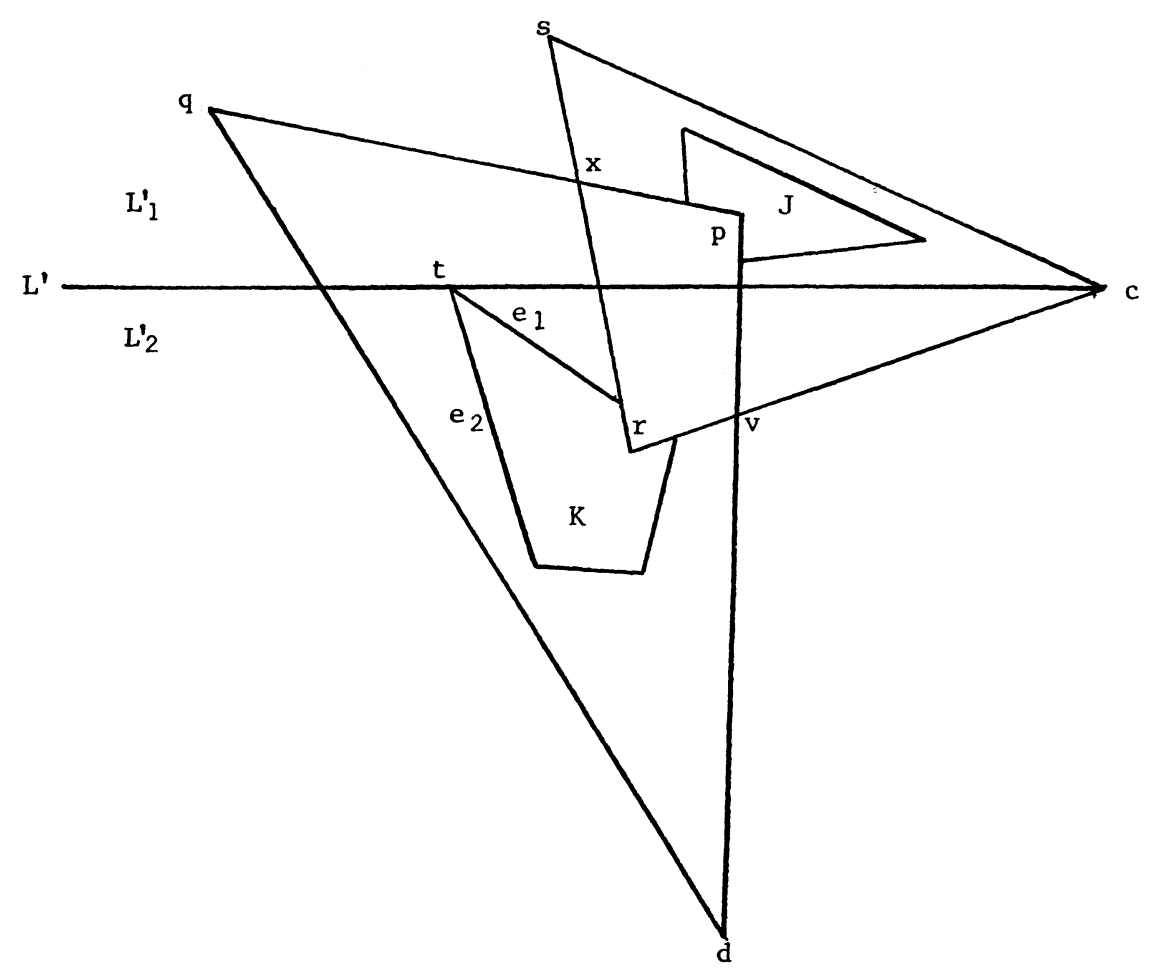

Figure 2

the points $c<p<x<q$. Then $p, q \in$ bdry $S$, neither is seen by $c$, so $d$ sees via $S$ both $p$ and $q$. Notice that $d \notin L(c, x)$ since $d$ cannot see $x$. Similarly, choose a segment at $x$ in $S \cap L(d, x)$ having maximal length, and let $r$ and $s$ denote its endpoints, $d<r<x<s$. Then point $c$ sees via $S$ both $r$ and $s$. (See Figure 2.)

Since points $c, d, x$ are not collinear, they determine a plane $P$ in $R^{3}$. In the next part of our proof, we restrict our attention to $P$. Since $[d, x] \nsubseteq S$, there is a segment in $(d, r) \sim S$, and this segment lies in a bounded component $K$ of $P \sim S, K \subseteq$ rel int $\operatorname{conv}\{d, p, q\}$. Likewise, there is a segment in $(c, p) \sim S$ belonging to a bounded component $J$ of $P \sim S, J \subseteq \operatorname{relint} \operatorname{conv}\{c, s, r\}$. Letting $L(c, r) \cap L(d, p)=\{v\}$, it is not hard to show that $J$ and $K$ lie in opposite open halfplanes of $P$ determined by $L(v, x)$.

For future reference, observe that for any line $U$ from $c$ meeting $K$, $d \notin U, d$ cannot see via $S$ all points of bdry $K$ on the opposite side of $U$ from $d$, so $c$ sees via $S$ some of these points. Thus if line $U^{\prime}$ from $c$ supports conv $K$, by a convergence argument, $c$ sees via $S$ some point of $U^{\prime} \cap($ bdry $K)$. We will use this observation in the next part of the proof. 
Define line $L^{\prime}$ and associated point $t$ as follows: Clearly $L(c, v) \cap J$ $=\varnothing$. In case $L(c, v) \cap K \neq \varnothing$, let $L_{1}$ denote the open halfplane of $P$ determined by $L(c, v)$ and containing $J$. Let $L^{\prime}$ be the line from $c$ supporting conv $K$ at a point of $L_{1}$. Using our previous observation, $L^{\prime} \cap($ bdry conv $K)$ contains some point $t$ of bdry $K$ such that $[c, t] \subseteq S$. In case $L(c, v) \cap K=\varnothing$, rotate $L(c, v)$ about $c$ toward $d$ until bdry $K$ is met. Let $L^{\prime}$ be the corresponding rotated line. Again using our observation, there is some $t \in L^{\prime} \cap($ bdry conv $K) \cap$ (bdry $K$ ) with $[c, t] \subseteq S$. Of course, in each case $t$ may be chosen to be the furthest point from $c$ having the required property. Moreover, $[c, t] \cap J=\varnothing$, and we may label the open halfplanes of $P$ determined by $L^{\prime}$ so that $J \subseteq L_{1}^{\prime}$. Then $K \cup\{d\}$ lies in the opposite halfplane $L_{2}^{\prime}$.

Since $S$ is a finite union of polytopes, bdry $K$ is necessarily a simple closed polygonal curve in plane $P$. By our choice of $t$, clearly $t$ is a point of local convexity of cl $K$. Also, $t$ must be a vertex of bdry $K$, so bdry $K$ contains two edges $e_{1}$ and $e_{2}$ at $t$. Moreover, for an appropriate labeling of these edges, $e_{1} \subseteq \mathrm{cl} L_{2}^{\prime}, e_{2} \subseteq L_{2}^{\prime} \cup\{t\}$, and for any neighborhood $N$ of $t$ with (cl $K) \cap N$ convex, $K \cap N$ and $c$ lie in the same open halfplane of $P$ determined by $L\left(e_{2}\right)$.

Using Lemma 3, select a plane $H$ such that $H \cap P$ is a line containing $e_{2}, K \cap N \subseteq H_{1}$, and $A_{t} \subseteq \mathrm{cl} H_{2}$. Similarly, select plane $M$ for $e_{1}$ so that $K \cap N \subseteq M_{1}$ and $A_{t} \subseteq \operatorname{cl} M_{2}$. Recall that by our choice of $c$ and $d$, at least one of these points lies in $\mathrm{cl} A_{t} \subseteq \mathrm{cl} H_{2} \cap \mathrm{cl} M_{2}$. Since $c$ and $K \cap N$ are in the same open halfplane of $P$ determined by $L\left(e_{2}\right), c \in H_{1}$. This forces $d$ to belong to $\mathrm{cl} H_{2} \cap \mathrm{cl} M_{2} \cap P$. However, clearly $\mathrm{cl} H_{2} \cap$ cl $M_{2} \cap P \subseteq \operatorname{cl} L_{1}^{\prime}$, while $d \in L_{2}^{\prime}$. We have a contradiction, our supposition is false, and every point of $S$ must see via $S$ either $c$ or $d$. Hence $S$ is a union of two starshaped sets, and Theorem 1 is established.

THeOREM 2. For $k \geq 1$ and $d \geq 1$, let $\mathscr{F}(k, d)$ denote the family of all compact unions of $k$ (or fewer) starshaped sets in $R^{d}, \mathscr{C}(k, d)$ the subfamily of $\mathscr{F}(k, d)$ whose members are finite unions of d-polytopes. Then $\mathscr{C}(k, d)$ is dense in $\mathscr{F}(k, d)$, relative to the Hausdorff metric. Moreover, $\mathscr{F}(k, d)$ is closed, relative to the Hausdorff metric.

Proof. In the proof, $h$ will denote the Hausdorff metric on compact subsets of $R^{d}$. That is, if $(A)_{\delta}=\{x: \operatorname{dist}(x, A)<\delta\}$, then for $A$ and $B$ compact in $R^{d}, h(A, B)=\inf \left\{\delta: A \subseteq(B)_{\delta}\right.$ and $\left.B \subseteq(A)_{\delta}, \delta>0\right\}$.

To see that $\mathscr{C}(k, d)$ is dense in $\mathscr{F}(k, d)$, let $S \in \mathscr{F}(k, d)$. For an arbitrary $\delta>0$, we must find some $C$ in $\mathscr{C}(k, d)$ for which $h(S, C)<\delta$. Assume that each point of $S$ is visible via $S$ from one of $s_{1}, \ldots, s_{k}$. Form 
an open cover for $S$, using interiors of $d$-simplices whose diameters are at most $\delta / 2$. Using the compactness of $S$, reduce to a finite subcover, say \{int $\left.P_{j}: 1 \leq j \leq m\right\}$, where $P_{j}$ is a $d$-simplex. For $1 \leq i \leq k$, define $C_{i}=\bigcup\left\{\operatorname{conv}\left(s_{i} \cup P_{j}\right): s_{i}\right.$ sees via $S$ some point of $\left.P_{j}, 1 \leq j \leq m\right\}$. Certainly set $C \equiv C_{1} \cup \cdots \cup C_{k}$ is a union of $k$ starshaped sets as well as a finite union of $d$-polytopes. Thus $C \in \mathscr{C}(k, d)$.

Clearly $S \subseteq C$, so $S \subseteq(C)_{\delta}$. To see that $C \subseteq(S)_{\delta}$, let $x \in C \sim S$. Then $x \in \operatorname{conv}\left(s_{i} \cup P_{j}\right)$ for some $i$ and $j$. Moreover, for an appropriate $i$ and $j$, there is some $y^{\prime} \in P_{j} \cap S$ with $\left[s_{i}, y^{\prime}\right] \subseteq S$. If $x, s_{i}, y^{\prime}$ are collinear, then since $x \notin S, x$ must belong to $P_{j}$, and $\operatorname{dist}\left(x, y^{\prime}\right) \leq \delta / 2$. Thus $x \in(S)_{\delta}$. If $x, s_{i}, y$ are not collinear, assume $x \in\left[s_{i}, y\right]$ where $y \in P_{j}$, and let $x^{\prime}$ be the point of $\left[s_{i}, y^{\prime}\right]$ such that $\left[x, x^{\prime}\right]$ and $\left[y, y^{\prime}\right]$ are parallel. Then $x^{\prime} \in S$ and $\operatorname{dist}\left(x, x^{\prime}\right) \leq \operatorname{dist}\left(y, y^{\prime}\right) \leq \delta / 2$. Again $x \in(S)_{\delta}$. We conclude that $C \subseteq(S)_{\delta}, h(S, C)<\delta$, and $\mathscr{C}(k, d)$ is indeed dense in $\mathscr{F}(k, d)$.

Finally, to see that $\mathscr{F}(k, d)$ is closed, let $\left\{S_{i}\right\}$ be a sequence in $\mathscr{F}(k, d)$ converging to the compact set $S_{0}$, to show that $S_{0} \in \mathscr{F}(k, d)$ also. For convenience of notation, for $i \geq 1$, let $S_{i}$ be a union of $k$ starshaped sets whose compact kernels are $A_{i 1}, A_{i 2}, \ldots, A_{i k}$, respectively. Then by standard results concerning the Hausdorff metric [6], $\left\{A_{i 1}\right.$ : $i \geq 1\}$ has a subsequence $\left\{A_{i 1}^{\prime}\right\}$ converging to some compact convex set $A_{1}$. Pass to the associated subsequence $\left\{S_{i}^{\prime}\right\}$ of $\left\{S_{i}\right\}$, and repeat the argument for corresponding kernels $\left\{A_{i 2}^{\prime}\right\}$. By an obvious induction, in $k$ steps we obtain subsequences $\left\{A_{i 1}^{(k)}\right\},\left\{A_{i 2}^{(k)}\right\}, \ldots,\left\{A_{i k}^{(k)}\right\}$ converging to compact convex sets $A_{1}, \ldots, A_{k}$, respectively. It is a routine matter to show that $S_{0}$ is a union of $k$ or fewer compact starshaped sets having kernels $A_{1}, \ldots, A_{k}$.

THEOREM 3. Let $S$ be a compact union of $k$ starshaped sets in $R^{d}$, $k \geq 1, d \geq 3$. Then there is a sequence $\left\{S_{j}\right\}$ converging to $S$ (relative to the Hausdorff metric) such that each $S_{j}$ satisfies property $P_{k}$. That is, using the notation of Theorem 2, sets having property $P_{k}$ are dense in $\mathscr{F}(k, d)$.

Proof. As in the proof of Theorem 2, $h$ will denote the Hausdorff metric on compact subsets of $R^{d}$. For any $\delta>0$, we must find some $C$ having property $P_{k}$ for which $h(S, C)<\delta$.

Assume that each point of $S$ is visible via $S$ from one of the distinct points $s_{1}, \ldots, s_{k}$. Form an open cover for $S$ using spheres of radius $\delta / 4$, centered at points of $S$. Reduce to a finite subcover, and choose the center of each sphere. Say these centers are the points $t_{1}, \ldots, t_{m}$. Partition 
$\left\{t_{1}, \ldots, t_{m}\right\}$ into $k$ subsets $V_{1}, \ldots, V_{k}$ such that the following is true: If $t \in V_{i}$, then $s_{i}$ is a point of $\left\{s_{1}, \ldots, s_{k}\right\}$ closest to $t$ with $\left[s_{i}, t\right] \subseteq S$. Define $T_{i}=\bigcup\left\{\left[s_{i}, t\right]: t \in V_{i}\right\}$. Observe that $s_{i} \notin T_{j}$ for $i \neq j$ : Otherwise, $s_{i} \in\left(s_{j}, t\right]$ for some $t \in V_{j},\left[s_{i}, t\right] \subseteq\left(s_{j}, t\right] \subseteq S$, and $s_{i}$ would be closer to $t$ than $s_{j}$ is to $t$, impossible by the definition of $V_{j}$.

In case the sets $T_{1}, \ldots, T_{k}$ are pairwise disjoint, let $T_{i}^{\prime}=T_{i}, 1 \leq i \leq k$, and define $T$ to be their union. Otherwise, suppose $T_{1}$ meets $T_{2} \cup \cdots \cup T_{k}$. Then for some point in $V_{1}$, call it $t_{1}$ (for convenience of notation), $\left(s_{1}, t_{1}\right.$ ] meets $T_{2} \cup \cdots \cup T_{k}$. Using the facts that each $T_{i}$ set is a finite union of edges at $s_{i}, s_{1} \notin T_{2} \cup \cdots \cup T_{k}$, and $d \geq 3$, it is not hard to show that there exists an edge $\left[s_{1}, t_{1}^{\prime}\right]$ not collinear with $\left[s_{1}, t_{1}\right]$ such that $\left[s_{1}, t_{1}^{\prime}\right]$ is disjoint from $T_{2} \cup \cdots \cup T_{k}$ and $\operatorname{dist}\left(t_{1}, t_{1}^{\prime}\right)<\delta / 4$. Thus $h\left(\left[s_{1}, t_{1}\right],\left[s_{1}, t_{1}^{\prime}\right]\right)$ $<\delta / 4$, also. Repeating the procedure for each edge of $T_{1}$, in finitely many steps we obtain a new set $T_{1}^{\prime}$ starshaped at $s_{1}$ such that $T_{1}^{\prime}$ is disjoint from $T_{2} \cup \cdots \cup T_{k}$ and $h\left(T_{1}, T_{1}^{\prime}\right)<\delta / 4$.

Continuing the process for $T_{2}, \ldots, T_{k}$, by an obvious induction we obtain pairwise disjoint starshaped sets $T_{1}^{\prime}, T_{2}^{\prime}, \ldots, T_{k}^{\prime}$ with $h\left(T_{i}, T_{i}^{\prime}\right)<$ $\delta / 4,1 \leq i \leq k$. Define $T=T_{1}^{\prime} \cup \cdots \cup T_{k}^{\prime}$. Standard arguments reveal that

$$
h\left(S, T_{1} \cup \cdots \cup T_{k}\right)<\frac{\delta}{4}, \quad h\left(T_{1} \cup \cdots \cup T_{k}, T\right)<\frac{\delta}{4},
$$

and hence $h(S, T)<\delta / 2$.

Finally, we extend the sets $T_{1}^{\prime}, \ldots, T_{k}^{\prime}$ to finite unions of $d$-polytopes. define $m=\min \left\{h\left(T_{i}^{\prime}, T_{j}^{\prime}\right): i \neq j\right\}$. Using techniques from Theorem 2, select set $C \equiv C_{1} \cup \cdots \cup C_{k}$ in $\mathscr{C}(k, d)$ with $h\left(T_{i}, C_{i}\right)<\min \{\delta / 2, m / 2\}$ and with $s_{i} \in \operatorname{ker} C_{i}, 1 \leq i \leq k$. Since $h\left(T_{i}, C_{i}\right)<m / 2$, certainly the $C_{i}$ sets must be pairwise disjoint. Therefore, each boundary point of $C$ is clearly visible from some $s_{i}, 1 \leq i \leq k$, and $C$ has property $P_{k}$. Moreover,

$$
h(S, C) \leq h(S, T)+h(T, C)<\frac{\delta}{2}+\frac{\delta}{2}=\delta .
$$

Theorem 3 is established.

It is interesting to observe that while Theorem 3 holds when $d \geq 3$, it fails in the plane, as the following easy example reveals.

EXAmple 1. Let $S$ be the set in Figure 3. Then $S$ is a union of two starshaped sets with kernels $\{c\},\{d\}$, respectively. However, sets sufficiently close to $S$ fail to satisfy the clear visibility condition required for property $P_{2}$. 


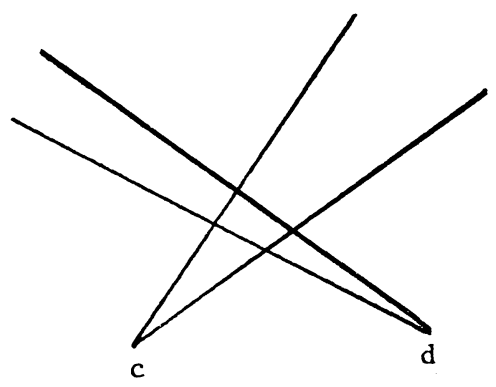

Figure 3

Finally, the characterization theorem for unions of two starshaped sets in $R^{3}$ is an easy consequence of our previous results.

CoROllary 1. Let $S \subseteq R^{3}$. Then $S$ is a compact union of two starshaped sets if and only if there is a sequence $\left\{S_{J}\right\}$ converging to $S$ (relative to the Hausdorff metric) such that each set $S_{j}$ satisfies property $P_{2}$.

Proof. The necessity follows immediately from Theorem 3. For the sufficiency, Theorem 1 implies that each set $S_{j}$ is a compact union of two starshaped sets in $R^{3}$. By Theorem 2, their limit $S$ is a compact union of two starshaped sets as well.

\section{REFERENCES}

[1] Marilyn Breen, Clear visibility and unions of two starshaped sets in the plane, Pacific J. Math., 115 (1984), 267-275.

[2] _ Points of local nonconvexity, clear visibility, and starshaped sets in $R^{d}$, J. Geometry, 21 (1983), 43-52.

[3] M. A. Krasnosel'skii, Sur un critère pour qu' un domaine soit étoilé, Math. Sb., 19 (61) (1946), 309-310.

[4] J. F. Lawrence, W. R. Hare, Jr. and John W. Kenelly, Finite unions of convex sets, Proc. Amer. Math. Soc., 34 (1972), 225-228.

[5] Steven R. Lay, Convex Sets and Their Applications, John Wiley, New York, 1982.

[6] S. Nadler, Hyperspaces of Sets, Marcel Dekker, Inc., New York, 1978.

[7] F. A. Valentine, Convex Sets, McGraw-Hill, New York, 1964.

Received November 21, 1985 and in revised form August 21, 1986.

UNIVERSITY OF OKLAHOMA

NORMAN, OK 73019 


\section{PACIFIC JOURNAL OF MATHEMATICS EDITORS}

\author{
V. S. VARADARAJAN \\ (Managing Editor) \\ University of California \\ Los Angeles, CA 90024 \\ HERBERT Clemens \\ University of Utah \\ Salt Lake City, UT 84112 \\ R. FINN \\ Stanford University \\ Stanford, CA 94305
}

HERMANN FLASCHKA

University of Arizona

Tucson, AZ 85721

RAMESH A. GANGOLLI

University of Washington

Seattle, WA 98195

VAughan F. R. JONES

University of California

Berkeley, CA 94720

ROBION KIRBY

University of California

Berkeley, CA 94720
C. C. MoOrE

University of California

Berkeley, CA 94720

H. SAMELSON

Stanford University

Stanford, CA 94305

HAROLD STARK

University of California, San Diego

La Jolla, CA 92093

\section{ASSOCIATE EDITORS}
R. AREnS
E. F. BECKENBACH
B. H. NEUMANN
F. WOLF
K. YOSHIDA (1906-1982)

\section{SUPPORTING INSTITUTIONS}

UNIVERSITY OF ARIZONA

UNIVERSITY OF BRITISH COLUMBIA

CALIFORNIA INSTITUTE OF TECHNOLOGY

UNIVERSITY OF CALIFORNIA

MONTANA STATE UNIVERSITY

UNIVERSITY OF NEVADA, RENO

NEW MEXICO STATE UNIVERSITY

OREGON STATE UNIVERSITY
UNIVERSITY OF OREGON UNIVERSITY OF SOUTHERN CALIFORNIA STANFORD UNIVERSITY UNIVERSITY OF HAWAII UNIVERSITY OF TOKYO UNIVERSITY OF UTAH WASHINGTON STATE UNIVERSITY UNIVERSITY OF WASHINGTON 


\section{Pacific Journal of Mathematics}

\section{Vol. 128, No. $1 \quad$ March, 1987}

Anthony Peter Bahri and Peter Gilkey, The eta invariant, $\operatorname{Pin}^{c}$ bordism, and equivariant $\operatorname{Spin}^{c}$ bordism for cyclic 2-groups $\ldots \ldots \ldots \ldots \ldots \ldots 1$

Friedrich-Wilhelm Bauer, Extensions of generalized homology theories . . . 25

Marilyn Breen, A characterization theorem for compact unions of two

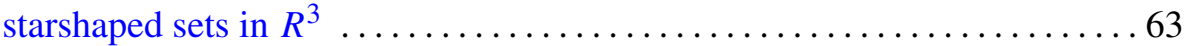

Boniface Ihemotuonye Eke, Special generating sets of purely inseparable extension fields of unbounded exponent $\ldots \ldots \ldots \ldots \ldots \ldots \ldots \ldots \ldots \ldots$

Robert William Gilmer, Jr. and William James Heinzer, Jónsson $\omega_{0}$-generated algebraic field extensions $\ldots \ldots \ldots \ldots \ldots \ldots \ldots \ldots \ldots . . .61$

Guido Lupacciolu, Holomorphic continuation in several complex variables

Douglas C. McMahon, Jaap C. S. P. van der Woude and Ta-Sun Wu,

Connectedness related to almost periodicity of compositions of flow

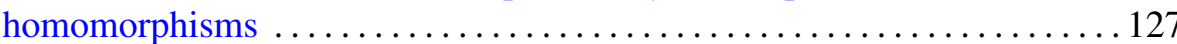

Sophocles Mercourakis, Some characterizations of analytic metric spaces

Peter Frederick Stiller, The Picard numbers of elliptic surfaces with many

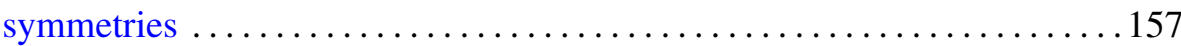

David J. Winter, Reducible complements of Lie algebra radicals ........ 191 Rade Živaljević, On a cohomology theory based on hyperfinite sums of microsimplexes 